\title{
Anharmonic and Quantum Fluctuations in Molecular Crystals: A First-Principles Study of the Stability of Paracetamol
}

\author{
Mariana Rossi, ${ }^{*}$ Piero Gasparotto*, and Michele Ceriotti ${ }^{\dagger}$ \\ Laboratory of Computational Science and Modeling, Institute of Materials, \\ École Polytechnique Fédérale de Lausanne, 1015 Lausanne, Switzerland
}

(Dated: July 6, 2021)

\begin{abstract}
Molecular crystals often exist in multiple competing polymorphs, showing significantly different physico-chemical properties. Computational crystal structure prediction is key to interpret and guide the search for the most stable or useful form: A real challenge due to the combinatorial search space, and the complex interplay of subtle effects that work together to determine the relative stability of different structures. Here we take a comprehensive approach based on different flavors of thermodynamic integration in order to estimate all contributions to the free energies of these systems with density-functional theory, including the oft-neglected anharmonic contributions and nuclear quantum effects. We take the two main stable forms of paracetamol as a paradigmatic example. We find that anharmonic contributions, different descriptions of van der Waals interactions, and nuclear quantum effects all matter to quantitatively determine the stability of different phases. Our analysis highlights the many challenges inherent in the development of a quantitative and predictive framework to model molecular crystals. However, it also indicates which of the components of the free energy can benefit from a cancellation of errors that can redeem the predictive power of approximate models, and suggests simple steps that could be taken to improve the reliability of $a b$ initio crystal structure prediction.
\end{abstract}

PACS numbers: 81.30.Hd, 61.50.Lt, 62.20.x, 71.15.m

Many compounds of biological and pharmaceutical importance crystallize as molecular crystals. Thus, predicting the stability of different polymorphs is of paramount importance, as the crystal structure can affect the solubility and pharmacokinetics of drugs [1,2]. In fact, there have been several incidents with drugs having to be recalled [3], production plants having to be halted [4], or patents being disputed [5] for reasons connected to the drug's polymorphism. In this field, the synergy between experiments and computational crystal structure prediction (CSP) promises to eliminate these inconveniences. However, the combinatorial complexity of the configuration space[6], as well as the need of evaluating relative stabilities of polymorphs with exquisite accuracy due to their very similar binding free energies, pose formidable obstacles to achieving this goal. In fact, this challenge has been recognized by the community: The Cambridge Crystallographic Data Centre has launched a series of blind tests focused on molecular crystal predictions, in order to assess the predictive power of various modelling approaches [7, 8]. Here, we will show for the paradigmatic example of paracetamol that although an accurate potential energy is necessary for the reliable modelling of these structures, anharmonic (quantum) free energy contributions are just as important. The development of accurate methods to evaluate these terms will be one of the major challenges to enable application of CSP to larger and more flexible compounds.

In this work we take a comprehensive approach involving several different types of thermodynamic integration in order to evaluate the full ab initio binding free energies of molecular crystals, disentangling the many differ- (a)
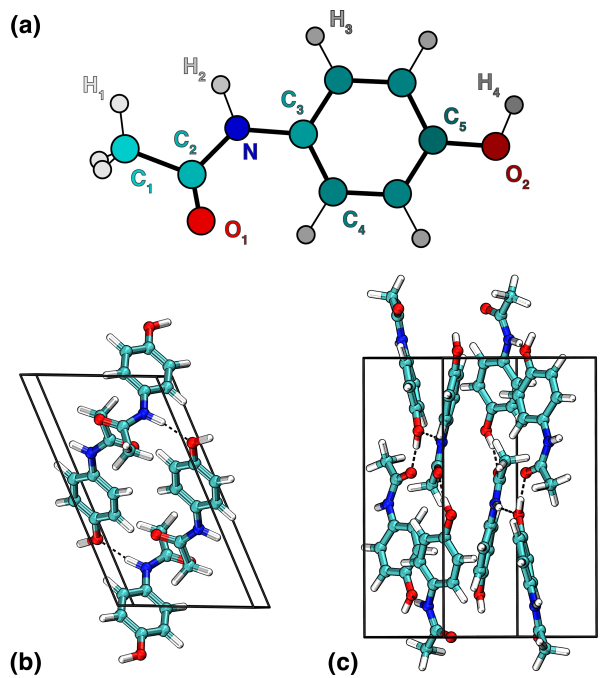

FIG. 1. (a) The paracetamol molecule including the color code that we use throughout the paper for each atom. Carbons are in shades of green, oxygens in shades of red, nitrogen is blue, and hydrogens are in shades of grey. The crystal structures of paracetamol in (b) monoclinic form I with four molecules per unit cell and (c) orthorhombic form II with eight molecules per unit cell.

ent contributions - harmonic, anharmonic, classical and quantum mechanical - that contribute to the free energies of these structures. We choose as an example Paracetamol (N-acetyl-p-aminophenol), a molecule of particular interest because of its wide use as an antipyretic and analgesic. We consider the monoclinic form I (fI) and the othorhombic form II (fII) $[9,10]$ (shown in Fig.1), which 
are the most common for this compound. They are predicted to be similarly energetically stable (differences are estimated to be between 10 and $60 \mathrm{meV} /$ molecule), with form I being favored at room temperature and pressure [11-14].

It is known that the relative stability of different forms can depend on the delicate interplay of qualitatively different physical effects, as has been recently demonstrated in the case of Aspirin [15, 16], where focus was given to van der Waals (vdW) forces and harmonic entropic terms. Other aspects that have received less attention so far are the contribution to free-energies that comes from nuclear quantum effects (NQEs), as well as the evaluation of temperature-related anharmonic effects, that have been proven necessary to assess the relative stability of ice polymorphs [17].

What we need to address is the magnitude of the contribution of these qualitatively different components to the absolute and relative free energies. Recent work on water and other hydrogen-bonded systems [18-22] has demonstrated that competing NQE in modes parallel and perpendicular to the hydrogen bonds partially compensate, reducing their net contribution to free energies. In compounds of pharmaceutical interest where polymorphs are almost isoenergetic (displaying different $\mathrm{H}$-bond networks), NQE can have an important impact. Anharmonic contributions can have markedly different magnitudes depending on the system in question - and for organic molecules containing soft modes, they are expected to play an important role [23]. As we will see, the magnitude of these contributions is strongly connected with the underlying potential energy surface, be it based on empirical potentials or on different electronic structure methods.

Our approach to disentangle these contributions relies on the combination of multiple thermodynamic transformations, as depicted schematically in Fig. 2, where we also introduce the notation we will use throughout this work to refer to the many components of the binding free energy. For harmonic free energies, it is sufficient to evaluate the vibrational frequencies for the system at hand and use textbook expressions for a simple harmonic oscillator. The difference between the value obtained with quantum and classical oscillators, $\delta_{\mathrm{QH}}^{\mathrm{CH}}$ gives a first idea of the importance of nuclear quantum fluctuations. Evaluating the anharmonic contributions with an ab initio potential is instead an authentic "tour de force", that required around two million CPU hours on a high-performance computing system, despite the fact that we deployed an array of acceleration techniques, as we will briefly summarize below (see also the SI).

We computed $\delta_{\mathrm{CA}}^{\mathrm{CH}}$ by thermodynamic integration from the Debye crystal to the full potential [24]. Even when using a Debye reference, the integrand exhibits a nearsingularity when approaching the full potential, probably due to the presence, of near-zero frequency libra-

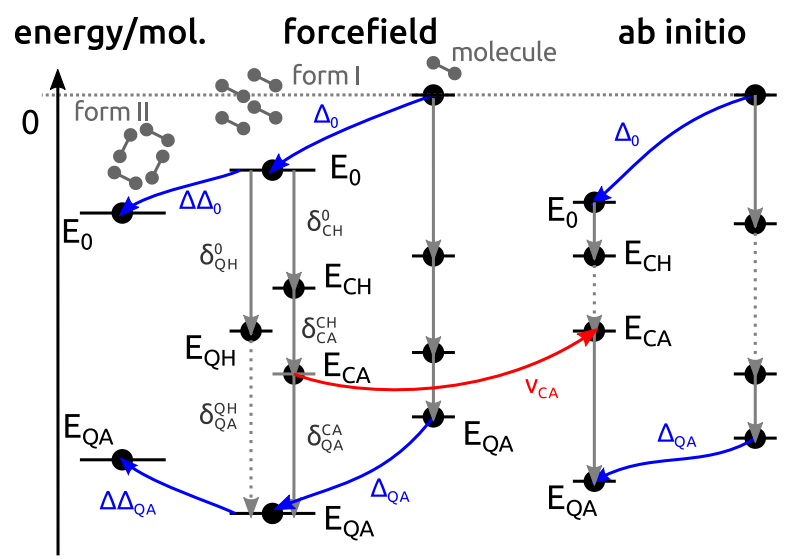

FIG. 2. A schematic representation of the many components to the cohesive free energy of molecular crystals. We indicate with $E_{\mathrm{X}}$ the (free) energy of a state at a given level of theory: $E_{0}$ indicates the bare potential energy, $E_{\mathrm{CH}}$ the classical freeenergy computed using a harmonic approximation, $E_{\mathrm{QH}}$ the quantum harmonic free-energy, $E_{\mathrm{CA}}$ the classical free-energy using the full anharmonic potential, $E_{\mathrm{QA}}$ the quantum anharmonic free-energy. Furthermore, we label $\delta_{\mathrm{Y}}^{\mathrm{X}}$ the energy difference between levels of theory (e.g. $\delta_{\mathrm{CA}}^{\mathrm{CH}}=E_{\mathrm{CA}}-E_{\mathrm{CH}}$ ), with $\Delta_{\mathrm{X}}$ the cohesive energy computed at a given level of theory (e.g. $\left.\Delta_{\mathrm{CA}}=E_{\mathrm{CA}}(\mathrm{fI}, \mathrm{FF})-E_{\mathrm{CA}}(\mathrm{mol}, \mathrm{FF})\right)$, with $\Delta \Delta_{\mathrm{X}}$ the relative stability of the two forms (e.g. $\Delta \Delta_{\mathrm{QA}}=$ $\left.\Delta_{\mathrm{QA}}(\mathrm{fI}, \mathrm{FF})-\Delta_{\mathrm{QA}}(\mathrm{fII}, \mathrm{FF})\right)$, and finally with $v_{\mathrm{X}}$ the variation in free-energy upon changing the potential energy surface.

tions of the methyl groups [25] - that instead appear as finite-frequency vibrations in the harmonic approximation (HA). To obtain a converged value for this term we had to (1) use a "poor man's self-consistent phonons" reference, in which we computed the Hessian using finite differences adapted to thermal fluctuations in the various directions; (2) use a highly non-uniform integration grid; (3) use a Padé interpolation to perform the integral, which can be motivated by considering the expression for the integrand in the case of two (different) harmonic reference potentials. Despite these stratagems, it would be still impractical to compute $E_{\mathrm{CA}}$ for an $a b$ initio (AI) potential. Instead, we first computed $E_{\mathrm{CA}}(\mathrm{FF})$, and then performed a thermodynamic integration switching from the force field $(\mathrm{FF})$ to the firstprinciples potential, which gives us access to $v_{\mathrm{CA}}$ (see Fig. 2) with a better-behaved integrand, and then computing $E_{\mathrm{CA}}(\mathrm{AI})=E_{\mathrm{CA}}(\mathrm{FF})+v_{\mathrm{CA}}$. The last term one needs to compute is the classical to quantum change in free energy, $\delta_{\mathrm{QA}}^{\mathrm{CA}}$. This can be obtained by performing a mass thermodynamic integration (MTI), evaluating the quantum kinetic energy of systems with scaled masses, as obtained from path integral molecular dynamics [21, 26, 27]. Again, in order to make this calculation feasible on an $a b$ initio potential, we had to combine all the tricks of the trade: We used (1) an integration variable that regularizes the integrand [27]; (2) a generalized Langevin equa- 


\begin{tabular}{c|ccc|ccc}
\hline \hline$[\mathrm{meV}]$ & fI $(\mathrm{FF})$ & fII $(\mathrm{FF})$ & $\Delta \Delta$ & fI $(\mathrm{AI})$ & fII $(\mathrm{AI})$ & $\Delta \Delta$ \\
\hline$\Delta_{0}$ & -1488 & -1487 & -1 & -1492 & -1489 & -3 \\
$\Delta_{\mathrm{CH}}$ & -1486 & -1508 & 22 & -1500 & -1487 & -13 \\
$\Delta_{\mathrm{CA}}$ & -1097 & -1120 & 23 & -1155 & -1118 & -38 \\
$\Delta_{\mathrm{QH}}$ & -1451 & -1474 & 23 & -1497 & -1479 & -18 \\
$\Delta_{\mathbf{Q A}}$ & $\mathbf{- 1 0 6 0}$ & $\mathbf{- 1 0 8 2}$ & $\mathbf{2 2}$ & $\mathbf{- 1 1 5 2}$ & $\mathbf{- 1 1 0 7}$ & $\mathbf{- 4 5}$ \\
\hline
\end{tabular}

TABLE I. Binding energies for form I and form II of paracetamol, in $\mathrm{meV} /$ molecule, computed at different levels of theory, using an empirical force field and $a b$ initio DFT$\mathrm{PBE}+\mathrm{D} 3$ simulations. $\Delta \Delta$ is the relative free-energy difference $\Delta(\mathrm{fI})-\Delta(\mathrm{fII})$.

tion to reach convergence of the quantum kinetic energy using only six path integral replicas [28]; (3) a multiple time step (MTS) integrator [29], alternating force evaluations with a more converged basis set and a cheaper, less-converged one. Details of this scheme and its convergence are discussed in the SI.

In order to perform the steps summarized above, we employed the i-PI program [30], with its recentlydeveloped MTS implementation [31], and its interfaces to LAMMPS [32] and CP2K [33, 34] in order to obtain the FF and DFT energies and forces, respectively. The FF parameters were generated through the SwissParam server [35], which is based on the Merck molecular force field (MMFF) [36]. For the DFT evaluations, we used the PBE [37] functional, combined with Goedecker-TeterHutter (GTH) pseudopotentials [38] and a TZV2P basis sets [33]. We included the D3 dispersion correction [39] for vdW interactions. The dynamics and vibrational frequency calculations were performed with only $\Gamma$ point sampling (see SI for a discussion on k-point sampling), and unless otherwise specified, all molecular dynamics simulations have been performed at $300 \mathrm{~K}$, using an optimal-sampling Generalized Langevin thermostat[40]. All free-energies will be quoted at this temperature. The crystal structure of both forms I and II were taken from the CCDC [41] and we kept the lattice parameters fixed at the experimental values, which were both measured at $300 \mathrm{~K}$. Optimizing the cell parameters did not change the relative energetics (see SI).

Table I reports the formation energies $\Delta_{\mathrm{X}}$ for the two phases at different levels of theory, as well as the difference in stability between form I and form II $(\Delta \Delta)$, based on an empirical force field (FF) and on ab initio (AI) calculations. Perhaps the most striking feature is that for both $\mathrm{FF}$ and $\mathrm{AI}$ calculations the two forms have a very similar binding energy $\Delta_{0}$, and that the key to understanding their relative stability lies in the quantum and finite-temperature contributions.

For the finite-temperature contributions, FF simulations consistently misrepresent the relative stability of the two forms, making form II more stable than form I. Although different contributions change very signifi- cantly the binding free energy - the most dramatic being the anharmonic classical free energy term, that destabilizes the crystal by almost $400 \mathrm{meV}$ - the changes are not reflected on the relative stability of the two forms, that is constant within $1 \mathrm{meV}$. AI data tells a different story. Form I is predicted to be the most stable structure, in agreement with experimental observations. Contrary to the FF case, the precise value of the free-energetic stability depends on a delicate balance between all the terms, with anharmonicity and quantum effects playing a crucial role. In this case, the classical, harmonic approximation would suffice to predict the most stable form. However, anharmonic and nuclear quantum effects contribute by as much as $30 \mathrm{meV} /$ molecule. We can thus infer that the FF simulations fail to grasp the differences in the physical effects brought by the different molecular stacking and H-bond pattern of these two forms.

NQE deserve a more in-depth analysis - both because they are typically neglected, and because they show strikingly different behavior in the FF and AI calculations. In the $\mathrm{FF}$, the quantum contributions to the free energies in the harmonic approximation $\left(\Delta_{\mathrm{QH}}-\Delta_{\mathrm{CH}}\right)$ amount to 35 and $33 \mathrm{meV}$ for forms I and II respectively. Including anharmonic effects $\left(\Delta_{\mathrm{QA}}-\Delta_{\mathrm{CA}}\right)$, these quantum contributions are slightly more positive (destabilizing the crystals), amounting to 37 and $38 \mathrm{meV}$ respectively. In the AI simulations, instead, the quantum contributions are much smaller and differ more significantly between the two forms. At the harmonic level quantum corrections amounts to only 2 and $8 \mathrm{meV}$ for forms I and II, and 3 and $11 \mathrm{meV}$ in the anharmonic case. In order to gain more insight into the role of quantum fluctuations, we analyzed the contribution of each atom in the paracetamol molecule to the binding quantum free energy. The PBE $+\mathrm{D} 3$ results (Figure 3 (a) and (b)) show that the small quantum contribution to the binding energy arises from a competition between quantum effects, as seen in many H-bonded systems [42, 43]. The $\mathrm{H}$-bonded hydrogen atom gives a significant stabilizing contribution, whereas most of the heavy atoms, as well as the non-bonding hydrogens, destabilize the crystalline phases. The stabilizing and destabilizing contributions from different atomic species sum up to small total values. Even if the details from the atomic contributions in the harmonic and anharmonic case are slightly different, these numbers are very small and similar, and we can only conclude that - at least in this case - the harmonic approximation is sufficient to grasp all the necessary quantum contributions to the free energies. It is interesting that form II is more destabilized by the quantum contributions than form I, and that those contributions make up for around a quarter of the free energy differences we observe between the two forms.

Performing a similar analysis for the FF simulations (Fig. 3(c)) explains why this inexpensive model differs so much from DFT. In contrast to the AI data, we find 


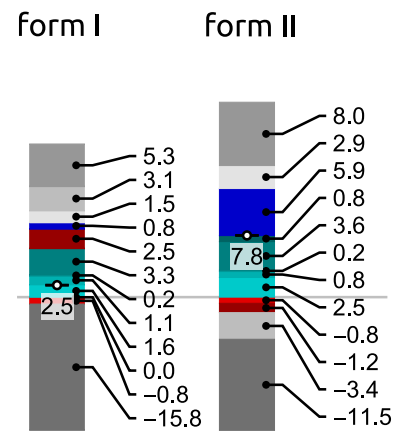

(a) HA PBE+D3

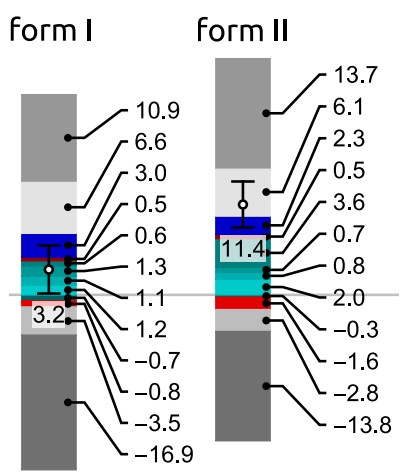

(b) MTI PBE+D3

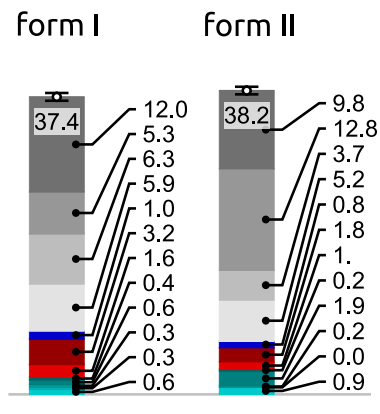

(c) MTI FF form I form II

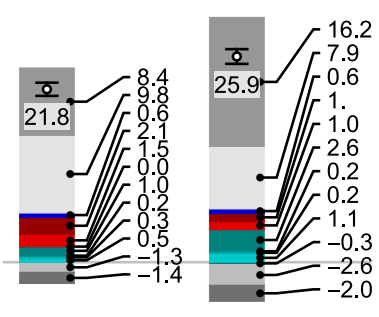

(d) MTI Mod. FF

FIG. 3. Bar plots showing the contribution of each atom (color coded as in Fig. 1(a)) and the total sum (black circles) of the quantum contributions to the free energies for form I and form II of paracetamol. All numbers are in meV/molecule. a) PBE+D3 functional, harmonic approximation $\Delta_{\mathrm{QH}}-\Delta_{\mathrm{CH}}$, b) PBE+D3 functional, mass thermodynamic integration $\Delta_{\mathrm{QA}}-\Delta_{\mathrm{CA}}$, c) orce field, mass thermodynamic integration $\Delta_{\mathrm{QA}}-\Delta_{\mathrm{CA}}$, d) force field with reparametrized anharmonic $\mathrm{OH}$, $\mathrm{NH}$, and $\mathrm{CH}$ stretches, mass thermodynamic integration $\Delta_{\mathrm{QA}}-\Delta_{\mathrm{CA}}$.

all contributions to be positive. The H-bonded atoms also engender a destabilizing effect upon binding. Inspired by the observation of the crucial role played by an anharmonic parameterization of the $\mathrm{OH}$ stretch in the description of NQE in water [19], we parametrized fourth order polynomial potentials for the $\mathrm{OH}, \mathrm{NH}$, and $\mathrm{CH}$ stretches based on our $\mathrm{PBE}+\mathrm{D} 3$ simulations for the isolated molecule (reported in the SI). We then recalculated the quantum anharmonic contributions with these modified parameters and functional form, which we show in Fig. 3(d). This simple modification makes the contribution of the $\mathrm{OH}$ and $\mathrm{NH}$ hydrogens negative (stabilizing), reducing $\Delta_{\mathrm{QA}}-\Delta_{\mathrm{CA}}$ by about $10 \mathrm{meV}$ for both forms. This effect can be explained based on the fact that stabilizing nuclear quantum contributions to the cohesive free energy arise due to modes that are red-shifted (softened) upon binding. The harmonic functional form of the $\mathrm{FF}$ does not allow the $\mathrm{OH}$ and $\mathrm{NH}$ stretches to soften sufficiently in the crystal forms, while even a simple anharmonic term allows for a significant degree of softening.

Although the main focus of this paper is a detailed analysis of free-energy corrections to the stability of molecular crystals, it is also necessary to discuss the role of the electronic-structure model. We refer the reader to Table II of the SI for the details of this analysis, where we compare different functionals, k-point meshes, and cell sizes. The most important points are that (i) Brillouin Zone sampling has a noticeable impact on binding energies, although, contrary to other examples [44], $\Gamma$ point sampling suffices for relative energetics. (ii) Exact exchange corrections seem to play a minor role in determining the relative stability of form I and form II. The PBE0 functional [45] with the Grimme D3 vdW correction $(\mathrm{PBE} 0+\mathrm{D} 3)$ predicts both forms to be isoenergetic
$\left(\Delta \Delta_{0}=0 \mathrm{meV}\right)$, similar to the $\mathrm{PBE}+\mathrm{D} 3$ prediction. (iii) Changing the form of the pairwise vdW correction has a more sizable effect. Using the Tkatchenko-Scheffler pairwise vdW correction [46] on top of the PBE functional (PBE+TS-vdW) stabilizes form II over form I by $\Delta \Delta_{0}=8 \mathrm{meV}$. (iv) Using a many-body vdW correction $[47,48]$ can change the energetics significantly, but the exact value depends subtly on supercell size. When using a large supercell, there is a small further stabilization of form II, yielding $\Delta \Delta_{0}=11 \mathrm{meV}$ (in good agreement with Ref. [14]). (v) Classical and quantum harmonic free energy corrections are relatively transferable if using pairwise dispersion energy corrections, but are not fully with many body dispersion. When considering a double unit cell for form I with the PBE+MBD functional, phonon contributions stabilize it by 3 (classical) and 10 (quantum) meV. However, if considering a single unit unit cell, form I is destabilized by 10 (classical) and 2 (quantum) meV. This observation is consistent with long range fluctuations described by this method that were reported in the literature $[16,49]$

The detailed analysis we performed for paracetamol underscores the grand challenge that is faced by efforts to predict the most stable polymorph from first principles [7]. Subtle, hard-to-compute terms such as the anharmonic free energy or NQE contribute by similar amounts to the overall energy balance, and any estimate that does not include the full package risks obtaining the right result for the wrong reason, or just a plain wrong result. To complicate things further, we observe a strong interplay between these free-energy terms and the underlying potential energy surface. A harmonic FF would incorrectly predict that the classical harmonic terms are enough to correct the baseline relative energy $\Delta \Delta_{0}$, whereas AI energetics show that anharmonic con- 
tributions are of paramount importance, and that quantum effects involve an almost complete cancellation between competing terms. The details of the AI calculation are also important: the kind of pairwise dispersion interactions and the use of many body dispersion corrections can change the relative stability of different polymorphs by tens of $\mathrm{meV}$. In this case, it appears that one can forgo expensive exact exchange calculations, but results on different materials suggest that this observation is not universal [15]. At the harmonic level, pairwise corrections predict form I to be more stable, in agreement with experiments [11]. MBD, which includes more physics and is generally more accurate, would apparently need the full anharmonic treatment in order to grasp this energetic balance - but the computation of these terms would be prohibitively expensive at this point.

The fact that anharmonic and quantum effects can be important also for a relatively simple molecule hints at the challenges that will be faced as CSP ventures into molecules with greater conformational flexibility. While great progress has been made towards reliable electronicstructure calculation of binding energies [44, 50, 51], the same attention should now be given to the evaluation of anharmonic and quantum free energy - to avoid painting an incomplete, possibly misleading picture. Future work shall investigate systematically these effects in different classes of molecular crystals, and benchmark different approximations to compute them inexpensively. Our study already provides hints at how to achieve predictive accuracy, without paying an unreasonable price. Computing anharmonic free energies through an indirect route that exploits integration from a FF reference greatly reduces the effort. It appears that even in the presence of competing quantum effects, $\delta_{\mathrm{QH}}^{\mathrm{CH}}$ gives a good estimate of the anharmonic quantum correction $\delta_{\mathrm{QA}}^{\mathrm{CA}}$, provided that the underlying potential energy surface can capture the environment-dependent softening of H-bonds. In that respect, augmenting empirical force fields to include anharmonic corrections to the bond energies could increase their reliability in predicting the impact of NQE. The path to an ab initio prediction of the stability of complex molecular crystals is ripe with challenges, but the stakes are high. With the combined progress in searching complex structural landscapes, predicting accurately $\mathrm{vdW}$ interactions, and efficiently estimating classical and quantum free-energy contributions, this goal is getting near.

We would like to thank V. Kapil for assistance in the implementation of normal-mode analysis in i-PI. We acknowledge generous allocation of CPU time by CSCS under the project id s618. This research was (partly) supported by the NCCR MARVEL, funded by the Swiss National Science Foundation, the SNF project 200021-159896 and the MPG-EPFL Center for Molecular Nanoscience. MR acknowledges funding by the Max Planck Society.
* These two authors contributed equally to the work.

$\dagger$ michele.ceriotti@epfl.ch

[1] M. L. Cheney, N. Shan, E. R. Healey, M. Hanna, L. Wojtas, M. J. Zaworotko, V. Sava, S. Song, and J. R. Sanchez-Ramos, Crystal Growth \& Design 10, 394 (2010).

[2] J. Kipp, International Journal of Pharmaceutics 284, 109 (2004).

[3] "Rotigotine patch recalled due to drug crystallization," http: //www .medscape.com/viewarticle/572798, accessed: 2016-03-31.

[4] J. Bauer, S. Spanton, R. Henry, J. Quick, W. Dziki, W. Porter, and J. Morris, Pharmaceutical Research 18, 859.

[5] Certain Crystalline Cefadroxil Monohydrate, Inv. 33\%TA-293 (DIANE Publishing).

[6] G. M. Day, T. G. Cooper, A. J. Cruz-Cabeza, K. E. Hejczyk, H. L. Ammon, S. X. M. Boerrigter, J. S. Tan, R. G. Della Valle, E. Venuti, J. Jose, S. R. Gadre, G. R. Desiraju, T. S. Thakur, B. P. Van Eijck, J. C. Facelli, V. E. Bazterra, M. B. Ferraro, D. W. M. Hofmann, M. A. Neumann, F. J. J. Leusen, J. Kendrick, S. L. Price, A. J. Misquitta, P. G. Karamertzanis, G. W. A. Welch, H. A. Scheraga, Y. A. Arnautova, M. U. Schmidt, J. Van De Streek, A. K. Wolf, and B. Schweizer, Acta Crystallographica Section B: Structural Science 65, 107 (2009).

[7] D. A. Bardwell, C. S. Adjiman, Y. A. Arnautova, E. Bartashevich, S. X. M. Boerrigter, D. E. Braun, A. J. CruzCabeza, G. M. Day, R. G. Della Valle, G. R. Desiraju, B. P. van Eijck, J. C. Facelli, M. B. Ferraro, D. Grillo, M. Habgood, D. W. M. Hofmann, F. Hofmann, K. V. J. Jose, P. G. Karamertzanis, A. V. Kazantsev, J. Kendrick, L. N. Kuleshova, F. J. J. Leusen, A. V. Maleev, A. J. Misquitta, S. Mohamed, R. J. Needs, M. A. Neumann, D. Nikylov, A. M. Orendt, R. Pal, C. C. Pantelides, C. J. Pickard, L. S. Price, S. L. Price, H. A. Scheraga, J. van de Streek, T. S. Thakur, S. Tiwari, E. Venuti, and I. K. Zhitkov, Acta Cryst. B 67, 535 (2011).

[8] C. R. Groom, I. J. Bruno, M. P. Lightfoot, and S. C. Ward, Acta Cryst. B 72, 171 (2016).

[9] M. Haisa, S. Kashino, and H. Maeda, Acta Cryst. B 30, 2510 (1974).

[10] M. Haisa, S. Kashino, R. Kawai, and H. Maeda, Acta Cryst. B 32, 1283 (1976).

[11] P. Espeau, R. Céolin, J. luis Tamarit, M. Perrin, J.-P. Gauchi, and F. Leveiller, Journal of Pharmaceutical Sciences 94, 524 (2005).

[12] T. Beyer, G. M. Day, and S. L. Price, The Prediction, Morphology, and Mechanical Properties of the Polymorphs of Paracetamol, Vol. 123 (2001) pp. 5086-5094.

[13] M. A. Neumann and M.-A. Perrin, CrystEngComm 11, 2475 (2009).

[14] R. A. DiStasio, O. A. von Lilienfeld, and A. Tkatchenko, Proc. Natl. Acad. Sci. USA 109, 14791 (2012).

[15] N. Marom, R. A. DiStasio, V. Atalla, S. Levchenko, A. M. Reilly, J. R. Chelikowsky, L. Leiserowitz, and A. Tkatchenko, Angewandte Chemie International Edition 52, 6629 (2013).

[16] A. M. Reilly and A. Tkatchenko, Phys. Rev. Lett. 113, 055701 (2014).

[17] E. A. Engel, B. Monserrat, and R. J. Needs, Phys. Rev. 
X 5, 021033 (2015).

[18] X.-Z. Li, B. Walker, and A. Michaelides, Proc. Natl. Acad. Sci. USA 108, 6369 (2011).

[19] T. E. Markland and B. J. Berne, Proc. Natl. Acad. Sci. USA 109, 7988 (2012).

[20] R. H. McKenzie, C. Bekker, B. Athokpam, and S. G. Ramesh, J. Chem. Phys. 140, 174508 (2014).

[21] M. Rossi, W. Fang, and A. Michaelides, J. Phys. Chem. Letters 6, 4233 (2015).

[22] M. Ceriotti, W. Fang, P. G. Kusalik, R. H. McKenzie, A. Michaelides, M. A. Morales, and T. E. Markland, Chem. Rev. 116, 7529 (2016).

[23] M. Rossi, M. Scheffler, and V. Blum, J. Phys. Chem. B 117, 5574 (2013).

[24] S. Habershon and D. E. Manolopoulos, Phys. Chem. Chem. Phys. 13, 19714 (2011).

[25] N. Tsapatsaris, B. a. Kolesov, J. Fischer, E. V. Boldyreva, L. Daemen, J. Eckert, and H. N. Bordallo, Molecular Pharmaceutics 11, 1032 (2014).

[26] W. Fang, J. Chen, M. Rossi, Y. Feng, X.-Z. Li, and A. Michaelides, J. Phys. Chem. Letters 7, 2125 (2016).

[27] M. Ceriotti and T. E. Markland, J. Chem. Phys. 138, 014112 (2013).

[28] M. Ceriotti and D. E. Manolopoulos, Phys. Rev. Lett. 109, 100604 (2012).

[29] M. Tuckerman, B. J. Berne, and G. J. Martyna, J. Chem. Phys. 97, 1990 (1992).

[30] M. Ceriotti, J. More, and D. E. Manolopoulos, Comp. Phys. Comm. 185, 1019 (2014).

[31] V. Kapil, J. VandeVondele, and M. Ceriotti, J. Chem. Phys. 144, 054111 (2016).

[32] S. Plimpton, J. Comp. Phys. 117, 1 (1995).

[33] "CP2K," http://www.cp2k.org.

[34] J. VandeVondele, M. Krack, F. Mohamed, M. Parrinello,
T. Chassaing, and J. Hutter, Comp. Phys. Comm. 167, 103 (2005).

[35] V. Zoete, M. A. Cuendet, A. Grosdidier, and O. Michielin, J. Comp. Chem. 32, 2359 (2011).

[36] T. A. Halgren, J. Comp. Chem. 17, 490 (1996).

[37] J. P. Perdew, K. Burke, and M. Ernzerhof, Phys. Rev. Lett. Phys. Rev. Lett. (USA), 77, 3865 (1996).

[38] S. Goedecker, M. Teter, and J. Hutter, Phys. Rev. B 54, 1703 (1996).

[39] S. Grimme, J. Antony, S. Ehrlich, and H. Krieg, J. Chem. Phys. 132, 154104 (2010).

[40] M. Ceriotti, G. Bussi, and M. Parrinello, J. Chem. Theory Comput. 6, 1170 (2010).

[41] F. H. Allen, Acta Cryst. B 58, 380 (2002).

[42] X.-Z. Li, B. Walker, and A. Michaelides, Proc. Natl. Acad. Sci. USA 108, 6369 (2011).

[43] M. Rossi, W. Fang, and A. Michaelides, J. Phys. Chem. Letters 6, 4233 (2015).

[44] J. Nyman and G. M. Day, CrystEngComm 17, 5154 (2015).

[45] C. Adamo and V. Barone, J. Chem. Phys. 110, 6158 (1999).

[46] A. Tkatchenko and M. Scheffler, Phys. Rev. Lett. 102, 073005 (2009).

[47] A. Tkatchenko, R. A. DiStasio, R. Car, and M. Scheffler, Phys. Rev. Lett. 108, 236402 (2012).

[48] A. Tkatchenko, A. Ambrosetti, and R. A. DiStasio, J. Chem. Phys. 138, 074106 (2013).

[49] A. Ambrosetti, N. Ferri, R. A. DiStasio, and A. Tkatchenko, Science 351, 1171 (2016).

[50] L. Kronik and A. Tkatchenko, Acc. Chem. Res. 47, 3208 (2014).

[51] J. G. Brandenburg and S. Grimme, J. Phys. Chem. Letters 5, 1785 (2014). 